\title{
An Architecture of Energy Efficient Restroom by Making Zero Standby Power
}

\author{
Karen Shibasaki, Sota Kono, and Akira Yamawaki* \\ Kyushu Institute of Technology, 1-1 Sensui, Tobata, Kitakyushu 804-8550, Japan \\ *Corresponding Author: yama@ecs.kyutech.ac.jp
}

\begin{abstract}
Electronic products need the standby power consumption to stay the standby mode to reduce the total power consumption. Although the standby power is smaller than the active power consumption, all electronic products consume the standby power which is comparative to the output of 1.6 nuclear power plants annually in Japan. The toilet is one of the standby power hungry products. The standby power consumption of all toiles used in home in Japan is equal to 18 billion yen. This paper proposes an architecture of energy efficient restroom by making zero standby power. We develop a prototype hardware and demonstrate the actual execution of our proposal.
\end{abstract}

Keywords: window security monitor, light sensor, wireless.

\section{Introduction}

To reduce the total power consumption, the electronic products often prepare the standby mode making the power consumption significant smaller than the active mode. Although the standby power is smaller than the active power consumption, all electronic products consume the standby power which is comparative to the output of 1.6 nuclear power plants annually in Japan ${ }^{(1)}$. The toilet is one of the standby power hungry products. The standby power consumption of all toilets used in home in Japan is equal to 18 billion yen. Including the public toilets in shopping malls, business buildings, amusement parks, and so no, the standby power consumed by all toilets in Japan seems to be a considerable problem to be solved.

To make the standby power consumption zero, we have proposed the power supply organization with zero standby power ${ }^{(2)}$. This paper proposes an architecture of energy efficient restroom employing our power supply with zero standby power consumption. We develop a prototype hardware and confirm the actual execution of our proposal.

The rest of paper is organized as follows. Section 2 shows the architecture of proposed toilet and its execution. Section 3 depicts the prototype hardware to verify the actual execution of our proposal. Section 4 shows the experimental results and discusses them. Finally, Section 5 concludes this paper and indicates the future work.

\section{Architecture and Execution}

\subsection{Toilet Architecture}

Fig. 1 shows the architecture of the proposed toilet. The remote controller attached to the wall or the side of the toilet bowls controls the functions of the toilet bowls. The main

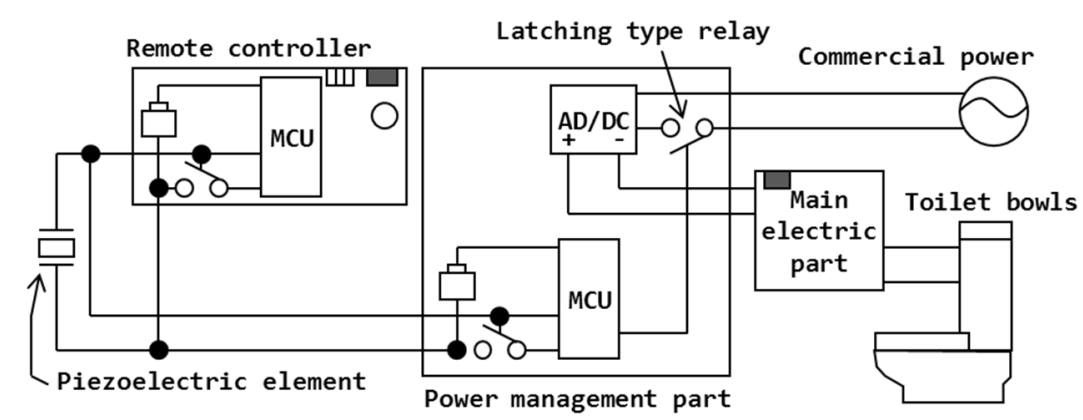

Fig. 1. Toilet Architecture 


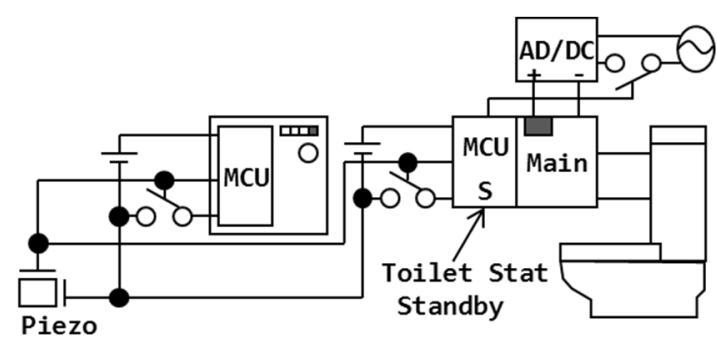

(a) Standby mode with zero power consumption

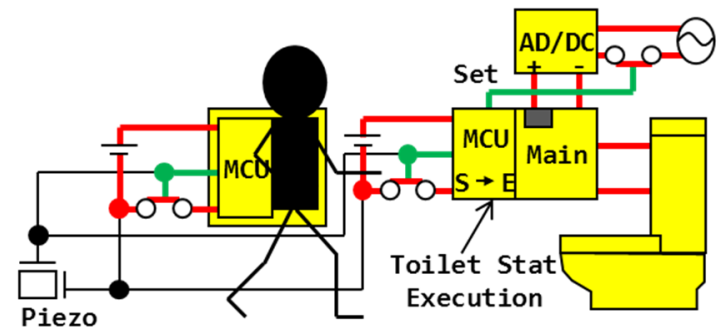

(c) Waking up mode with activation of Toilet bowls

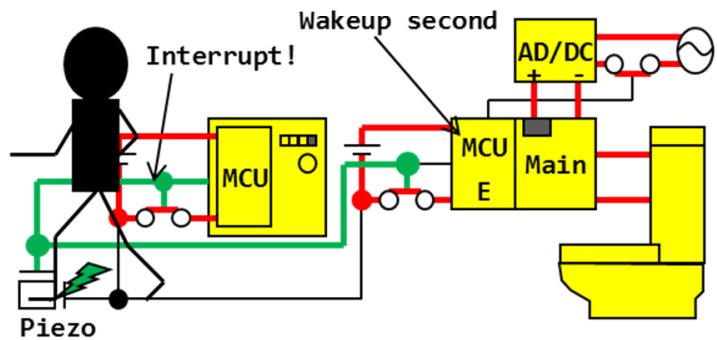

(e) Exiting mode with interrupt and waking up again

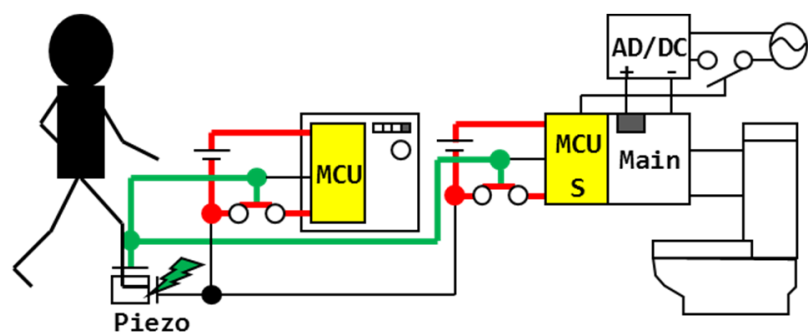

(b) Entering mode with activation of MCUs

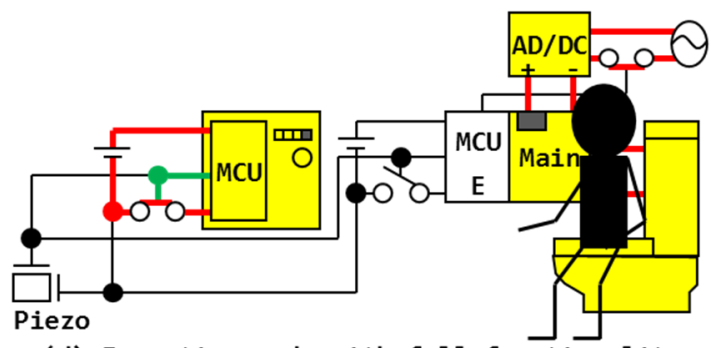

(d) Execution mode with full functionality

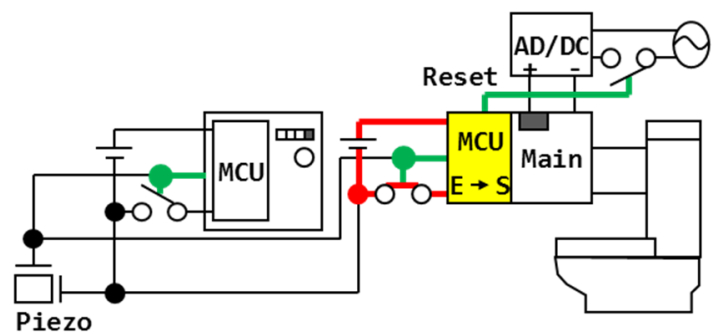

(f) Going standby mode with turning off power

Fig. 2. Execution Snapshot of Proposed Toilet

electronic part has functionalities such as automatic seat warmer, lid moving, showering, flushing, and so on. It is equipped to the toilet bowls.

Conventionally, the toilet consumes the standby power to perform the seat warming, water warming, human sensing, $\mathrm{AD} / \mathrm{DC}$ converting, and so on. The battery of the remote controller is also consumed constantly.

To cut all of them, the proposed architecture has several power switches as shown in Fig. 1, which disconnect the ground lines of the microcontrollers and the $\mathrm{AC}$ line in standby. The power switch attached to the remote controller is a MOSFET transistor to eliminate the standby power of the remote controller. The power switch attached to the power management part is also a MOSFET transistor. The power switch existing on the $\mathrm{AC}$ line is a latching type relay.

In addition, the piezoelectric element is equipped as the human sensor and the energy harvester to turning on the power transistor. The piezoelectric element is placed to the ground near the entrance of the restroom where the user can tread on or the door edge where the piezo element can be flipped by the door opening and closing.

\subsection{Execution of Proposed Architecture}

Fig. 2 shows the execution snapshots of the proposed toilet architecture.

Standby mode in Fig. 2 (a): All circuits in the toilet has no closed loop in standby mode. Thus, the consumed current becomes zero; the standby power is zero. The conventional toilet has to consume some power to detect human entering the restroom while our proposal never consumes any power but can detect human by using the electromotive force generated by the piezoelectric element.

Entering mode in Fig. 2 (b): When a human is entering the restroom, the piezoelectric element is treaded on and generates some electromotive force. The generated electromotive force turns on the power transistors attached to the ground lines of microcontrollers. As a result, the microcontrollers wake up because the closed loops appear from the plus to the minus of the battery.

Waking up mode in Fig. 2 (c): The microcontrollers are preparing to wake up the whole of toilet. Instead of the piezoelectric elements, the microcontrollers keep driving the power transistor to continue the execution. The microcontroller in the power management part connects the $\mathrm{AC}$ line by setting the latching relay. The $\mathrm{AD} / \mathrm{DC}$ converter starts the execution and supplies the DC power to the toilet bowls. As a result, the remote controller and the toilet bowls are going to the active mode. In this period, the seat and water 
warming can be completed by the rapid warming technology currently used.

Execution mode in Fig. 2 (d): Since the microcontroller in the remote controller continues to turn on the power transistor, the remote controller acts as the normal remote controller with full functionality. The microcontroller in the power management part disconnects the power transistor bridging the ground line and stores the toilet status (execution) in the non-volatile storage like an embedded flush memory. Thus, the power management part does not consume any power in the normal execution of the toilet bowls. The AC line is constantly connected by the latching relay previously set without any power to keep closing. As a result, the user can use the toilet with full functionality using the active power as equal to the conventional one.

Exiting mode in Fig. 2 (e): When the user finishes to use the toilet and exits the rest room, the piezoelectric element is treaded on again. Thus, the electromotive force is generated. In this period, the microcontroller in the remote controller uses it as the external interrupt turning off the power. The microcontroller in the power management part wakes up again. Since the toilet status previously stored is the execution, the microcontroller knows that the toilet bowls must be turned off.

Going standby mode in Fig. 2 (f): The microcontroller in the remote controller turns off its own power transistor in the interrupt service routine. That of the power management part resets the latching relay and modifies the status in the non-volatile storage to the standby. As a result, the proposed toilet goes to the standby mode shown in Fig. 2 (a).

\section{Prototype Hardware}

Fig. 3 shows the block diagram of the prototype hardware. To rapidly perform the preliminary experiments only to confirm the basic execution shown in Fig. 2, we imitated the remote controller and main electric part of the toilet as followings.

The remote controller has three buttons, red, green, and blue. Each button sends the square waves of $1 \mathrm{KHz}$ with $50 \%$ duty to the driver of IR LEDs. The numbers of pulses are 10, 20 and 30 for the red, green and blue button respectively.

The main electric part acquires the commands according to the irradiated pulses from the remote controller. The photodiode outputs the blinking pulses when receiving the irradiated light from the remote controller. The received blinking pulses are converted to a single long width pulse by the smoothing circuit. The main electric part decides which button is pushed by counting the width of the smoothed pulse and turns on the dedicated LED.

The power management part used the latching relay to store the toilet status (standby or execution, E/S\#). The relay switch used has two pole flipping by set/reset contradictory each other. One pole is used to bridging the AC line. Another

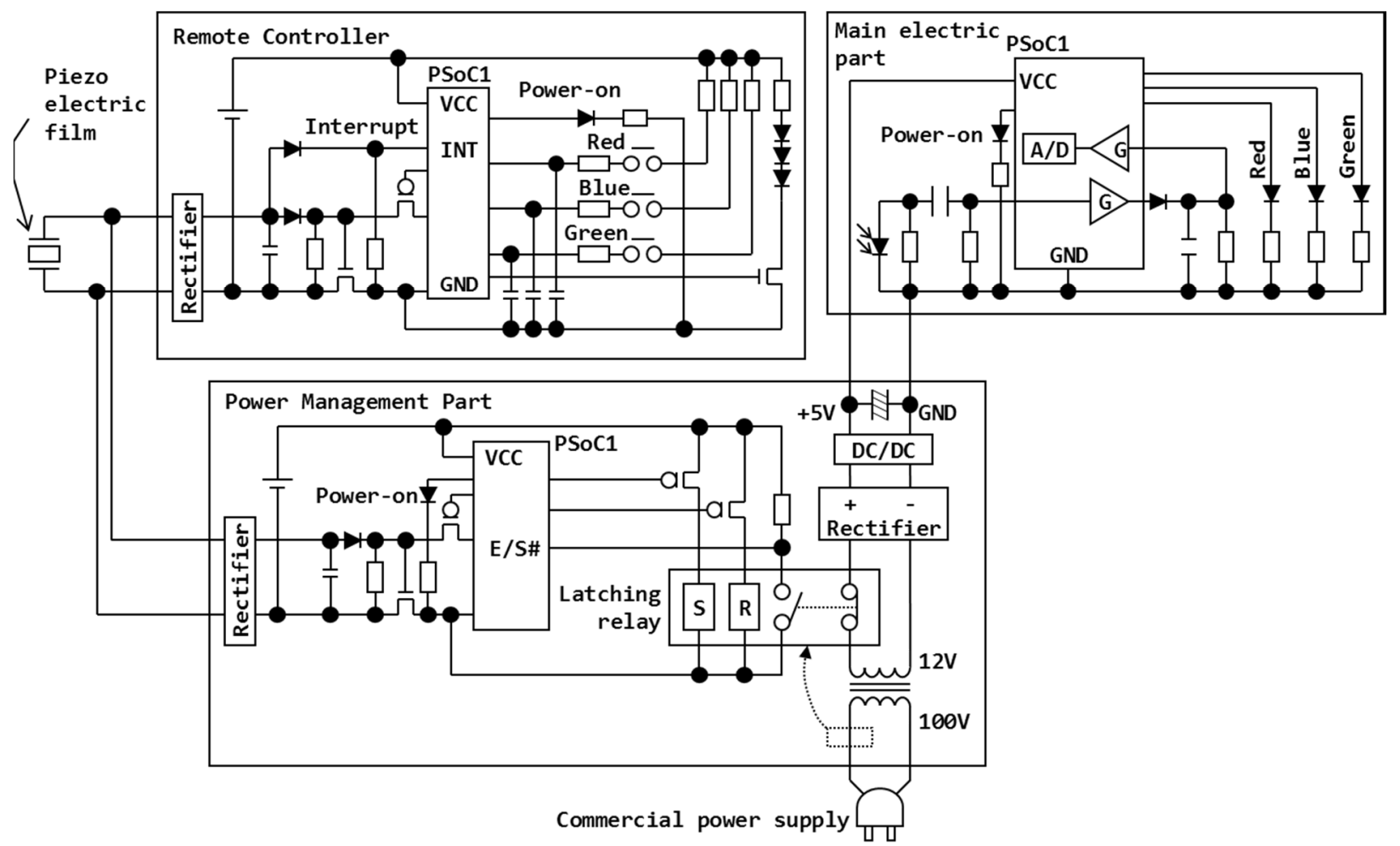

Fig. 3. Prototype Hardware 


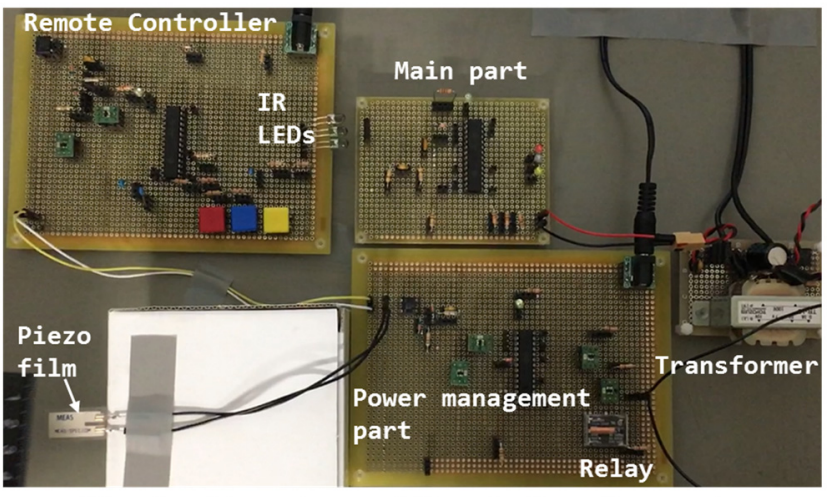

Fig. 4. Experimental Result: Standby mode

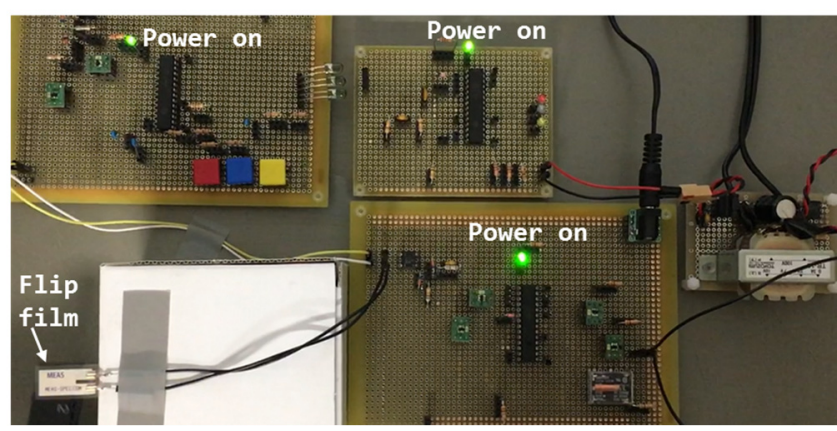

Fig. 5. Experimental Result: Entering and waking up
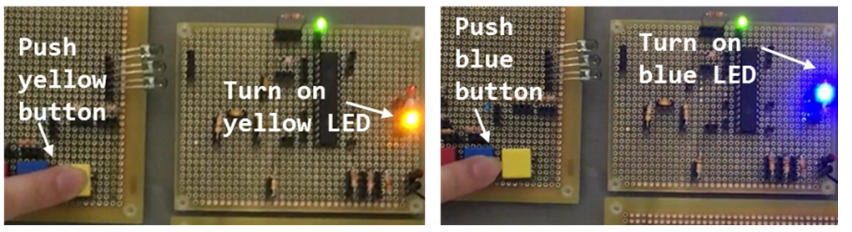

Fig. 6. Experimental Result: Execution mode

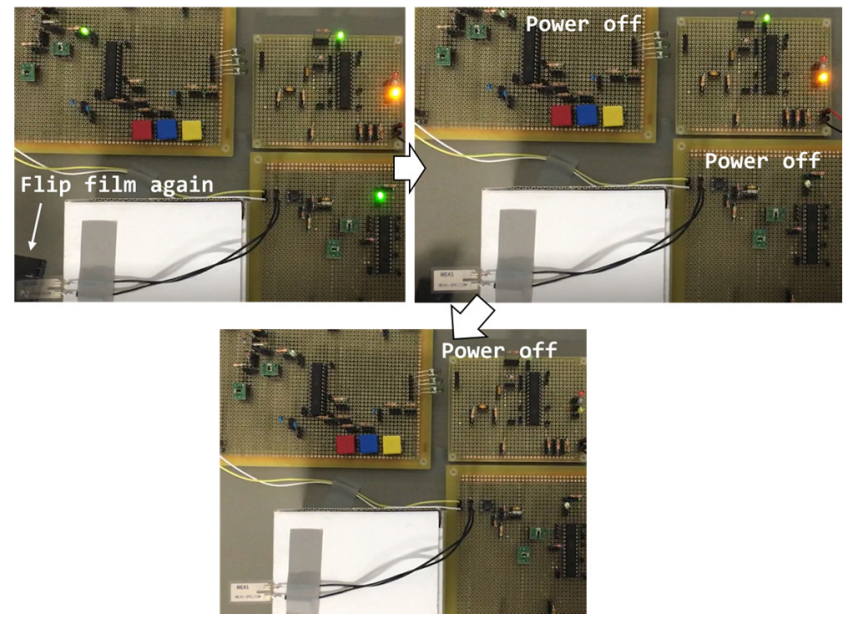

Fig. 7. Experimental Result: Going standby mode

is used as non-volatile storage to store the toilet status as mentioned above. Note that inherently the relay switch has to be placed into the $\mathrm{AC}$ line directly to the commercial power supply, where the dotted box shown in Fig. 3 exists. Due to safety of the experiment, we inserted the relay switch after the transformer from $100 \mathrm{~V}$ to $12 \mathrm{~V}$ in this experiment.

\section{Experiment}

Fig. 4-7 show the experimental results demonstrating the actual execution shown in Fig. 2.

Fig. 4 indicates that the prototype hardware stays at the standby mode and the power consumption becomes zero.

When flipping the piezoelectric film as shown in Fig. 5, the remote controller, the power management part and the main part are waken up and they indicate their own awakening by turning on the power-on LEDs.

The main part waken up is controlled by the remote controller waken up as shown in Fig. 6. When the user pushes the yellow / blue button on the remote controller, the main part turns on the yellow / blue LED as imitation of the toilet behavior.

Fig. 7 depicts the execution snapshots of going standby mode. When flipping the piezo film again, the interrupt occurs to the remote controller and the power management part is waken up again. The microcontroller on the remote controller turns off the power transistor in the interrupt routine. The remote controller quickly goes to the standby mode turning off the power-on LED. The power management part also quickly goes to the standby mode as well. In contrast, the main part is delayed to go to the standby mode because the power rail of the main part has the large capacitor at the output of the DC/ DC converter, which is discharged with some period. As a result, the proposed mechanism has gone to the standby mode correctly.

\section{Conclusions}

We have proposed the architecture of energy efficient restroom employing our power supply with zero standby power. We have developed a prototype hardware and confirmed the actual execution of our proposal. As future work, we will develop a real toilet employing our proposal and evaluate its practical power consumption and usability.

\section{References}

(1) Agency for Natural Resources and Energy, http://w ww.enecho.meti.go.jp/category/saving_and_new/savin $\mathrm{g} /$ general/report/ (2014) in Japanese.

(2) Akira Yamawaki and Seiichi Serikawa: "Remote P ower Switch to Make Standby Power of Infrared Remote Controlled Product Zero", IAENG Internati onal Journal of Computer Science, vol. 42, no. 2, pp.132-138 (2015). 